\title{
Imaging of the domain organization in sphingomyelin and phosphatidylcholine monolayers
}

\author{
Elmar Prenner ${ }^{\mathrm{a}, *}$, Gerlinde Honsek $^{\mathrm{a}}$, Dirk Hönig ${ }^{\mathrm{b}}$, \\ Dietmar Möbius $^{c}$, Karl Lohner ${ }^{\mathrm{d}}$ \\ ${ }^{a}$ Department of Biological Sciences, 2500 University Drive, University of Calgary, Calgary, Alberta T2N 1N4, Canada \\ b Nanofilm Technologie GmbH, Göttingen, Germany \\ ${ }^{c}$ Max-Planck-Institut für Biophysikalische Chemie, Göttingen, Germany \\ ${ }^{\mathrm{d}}$ Institute of Biophysics and X-ray Structure Research, Austrian Academy of Sciences, Graz, Austria \\ Received 14 July 2006; received in revised form 3 November 2006; accepted 13 November 2006 \\ Available online 21 November 2006
}

\begin{abstract}
The lateral organization of biomembranes has gained significant interest when the fluid mosaic model was challenged by the model of "lipid rafts". Several lipid classes like cholesterol and sphingolipids are considered to be essential for their formation. Here we investigate the lateral domain formation in binary mixtures of sphingomyelin and phosphatidylcholine. Both are major lipid components of lipoproteins and mammalian cell membranes at various molar ratios. Surface pressure-area isotherms and surface potential-area isotherms of monolayers composed of these lipids clearly indicated non-ideal mixing. In addition, Brewster angle microscopy provided a well-suited approach to image the formation of lateral domains. These images demonstrated that pure sphingomyelin forms very stable finger-like domains that exhibit a distinct internal organization suggesting an anisotropic orientation of the acyl side chains. Similar behavior was found for mixtures containing more than $60 \mathrm{~mol} \%$ sphingomyelin. With increasing content of phosphatidylcholine the domain size decreased and the surface pressure, where domain formation occurred, increased. At lower sphingomyelin content (30-60 mol\%) rather round-shaped, smaller domains were observed. Thus, the potential of sphingomyelin domains as potentially important building blocks for actual domains that could be building blocks for raft formation is suggested, even without the presence of cholesterol. In addition, these observations may suggest a role for the distinct molar ratio of these key lipids frequently found in physiologically relevant particles such as low and high density lipoproteins or the outer leaflet of the human erythrocyte membrane.
\end{abstract}

(C) 2006 Published by Elsevier Ireland Ltd.

Keywords: Brewster angle microscopy; Lipid packing; Lipid domain formation; Monolayer; Membrane mimetic system

Abbreviations: SM, sphingomyelin; PC, phosphatidylcholine; CHOL, cholesterol; POPC, 1-palmitoyl-2-oleoyl-phosphatidylcholine; BAM, Brewster angle microscopy; LDL, low density lipoprotein; HDL, high density lipoprotein

* Corresponding author. Tel.: +1 403220 7632; fax: +1 4032899311 .

E-mail address: eprenner@ucalgary.ca (E. Prenner).

\section{Introduction}

Glycerol-based phospholipids and sphingomyelins are among the most abundant lipids in mammalian plasma membranes. Within the last decade, sphingolipids have emerged as active participants in cell regulation since these lipids and their metabolites are involved in differentiation, cellular senescence or apoptosis (Ohanian and Ohanian, 2001). Furthermore, 
the fusion of some viruses is enhanced or dependent on sphingolipids being present in the target membrane (Van-Mau et al., 2000; Saez-Cirion et al., 2002).

Recently the classical fluid bilayer model was challenged by the concept of lipid islands termed "rafts" supported by findings of detergent insoluble membrane fragments enriched in certain lipids like sphingomyelin (SM) and cholesterol (CHOL) (Brown and London, 1998). The model of functional lipid rafts as outlined previously (Simons and Ikonen, 1997) suggested various biological functions for these moieties and thus emphasized the importance of lipid organization in various biological systems (see also e.g. Anderson and Jacobson, 2002; Harder, 2003; Simons and Vaz, 2004). A lipid domain structure for bilayers was earlier suggested from lateral diffusion studies of lipids in sarcoplasmic reticulum membranes that was shown to be area-limited (Laggner, 1981).

Biophysical investigations of model systems have made significant contributions to the understanding of lipid rafts (for recent reviews see Simons and Toomre, 2000; London, 2002). For example, Silvius and coworkers showed differential partitioning of SM into SM/CHOL domains (Wang and Silvius, 2000) and partitioning of lipidated peptides into ordered lipid domains (Wang et al., 2001). Many contributors to the field discussed a vital role for cholesterol in the formation and stability of such membrane rafts (Radhakrishnan et al., 2000; Xu et al., 2001; Veatch and Keller, 2003) and initial imaging of raft structures by atomic force microscopy was reported (Rinia et al., 2001; Giocondi et al., 2004). But interestingly, an attenuating effect of cholesterol on domain formation was also reported for renal brush border membranes (Milhiet et al., 2001). Moreover, phosphatidylcholine and sphingomyelin, characterized by the same headgroup but different backbone organization, are major components of the outer membrane layer of platelets and erythrocytes (Cesar et al., 1993) or the monolayer of lipoproteins (Barenholz and Gatt, 1982). They are abundant in different characteristic molar ratios like 70\% PC and 30\% SM for low density lipoprotein (LDL) (Sommer et al., 1992), about 55\% PC to $45 \%$ SM for the human erythrocyte membrane (Cesar et al., 1993 ) or about $15 \%$ PC and $85 \%$ SM in certain high density lipoprotein subclasses (HDL) (Barenholz and Gatt, 1982).

Therefore, our working hypotheseis has been that these obvious significant differences in the PC/SM ratio are not random, but will impact bilayer properties, and hence membrane functionality, as well as the monolayer properties in lipoproteins. In particular, we are interested in the possible existence of any SM domains, even without the interaction with cholesterol that is commonly seen to induce lateral demixing (Radhakrishnan et al., 2000). In this work we report on the mixing behaviour of two major mammalian lipids, PC and SM as deduced from the surface pressure and potential versus area isotherms. In addition, the formation of distinct SM domains as a function of PC admixture was imaged by Brewster angle microscopy. This technique, developed independently by two groups (Henon and Meunier, 1991; Hönig and Möbius, 1991), is an important imaging tool that allows the direct visualization of monolayers at the air/water interface without the addition of a potentially perturbing fluorescent probe.

\section{Materials and methods}

\subsection{Phospholipids}

1-Palmitoyl-2-oleoyl-phosphatidylcholine (POPC) and egg-sphingomyelin (SM), containing primarily $\mathrm{N}$-linked palmitate, were purchased from Avanti Polar Lipids (Alabama, USA). Purity of both lipids (>99\%) was checked by thin layer chromatography using $\mathrm{CHCl}_{3}: \mathrm{MeOH}: \mathrm{H}_{2} \mathrm{O}$ (75:25:6, v/v) as solvent, whereby only one single spot was detected. The exact amount of phospholipid stock solutions was determined according to the method of Bartlett (1959).

\subsection{Monolayer experiments}

Surface pressure-area $(\pi-A)$ isotherms were recorded using a customer-designed rectangular Teflon trough (Nanotechnology, MDT Corp., Moscow, Russia) thermostated at $25^{\circ} \mathrm{C}$. A moveable Teflon barrier compressed the monolayer with compression velocity between 0.07 and $0.12 \mathrm{~nm}^{2} /($ min molecule). A Wilhelmy balance ( $20 \mathrm{~mm}$ wide filter paper) was used to measure the surface pressure. All glass was cleaned with chromosulfuric acid and rinsed extensively with highly purified water to avoid any traces of impurities. The subphase was highly purified water, prepared with the Milli-RO4 + Milli-Q water purification system. The monolayers were prepared by spreading a defined volume of a $\mu \mathrm{M}$ lipid solution in chloroform (Baker analyzed HPLC grade) onto the aqueous subphase using a Hamilton syringe. The system was enclosed in a box in order to minimize water evaporation, to ensure high humidity, and to avoid trace pollution of the system. All values displayed are highly reproducible and represent the average of at least three experiments and the standard deviation is less than $1 \%$ for surface pressure and less and $5 \%$ for the surface potential values. 


\subsection{Analysis of isotherms}

The mixing behavior of the two lipid components was analyzed by calculating the mean molecular area $\left(A_{\pi}\right)$ (see Ali et al., 1994):

$A_{\pi}=X_{1}\left(A_{1}\right)_{\pi}+\left(1-X_{1}\right)\left(A_{2}\right)_{\pi}$

whereby $X_{1}$ is the mole fraction of component $1 .\left(A_{1}\right)_{\pi}$ and $\left(A_{2}\right)_{\pi}$ are the mean molecular areas of the two pure components at a given surface pressure. For ideal behavior, the variation of the mole fractions results in a linear relation. Compressibility data were calculated for pure lipid monolayers at a given surface pressure $\pi$ from the $\pi-A$ isotherm using (Keller, 2003):

$k_{\pi}=\left(-\frac{1}{A_{\pi}}\right)\left(\frac{\mathrm{d} A}{d_{\pi}}\right)_{\pi}$

According to Ali et al., the mean compressibility for the mixtures was calculated assuming ideal behavior analogously to the mean molecular areas except that the relative contribution of each lipid depends on the molecular area as well as on their molar ratio:

$k_{\pi}=-\frac{1}{A_{\pi}\left[\left(k_{1} A_{1}\right)_{\pi} X_{1}+\left(k_{2} A_{2}\right)_{\pi} X_{2}\right]}$

As data near the high and low-pressure limits are particularly sensitive to trace impurities (Ali et al., 1991), we constrained our analysis to the range between 10 and $35 \mathrm{mN} / \mathrm{m}$, the latter value being well below the collapse pressure of the respective films. The measured $\Delta V$-values are given by the difference of the potential of the monolayer and the potential of the pure water surface (Kuhn and Möbius, 1993) and were measured by using the vibrating plate method (for details see (Vogel and Möbius, 1988a)) in the same pressure range $(10-35 \mathrm{mN} / \mathrm{m})$. Analogously to (Eq. (1)) the average surface potential $(\Delta V)$ as a function of lipid composition provides information about the mixing behavior of the components. For a comparison with biomembranes, the values at higher pressure are more important since calculations proposed about $30-35 \mathrm{mN} / \mathrm{m}$ for the lateral membrane pressure (Marsh, 1996). In this range the $\Delta V-A$ behavior for single-component films is described by the classical Helmholz equation (Vogel and Möbius, 1988b):

$\varepsilon_{0} \Delta V A=\Delta \mu_{\perp}$

where $\varepsilon_{0}$ is the permittivity of the vacuum, $\Delta V$ the potential measured in $\mathrm{mV}, A$ the experimental mean molecular area $\left(\AA^{2} /\right.$ molecule) and $\Delta \mu_{\perp}$ is the normal component of the mean surface dipole moment expressed in units of Debye (D) (Vogel and Möbius, 1988b).

\subsection{Brewster angle microscopy (BAM)}

The BAM technique is based on the principle that p-polarized light, directed on a transparent substrate under the Brewster angle, is not reflected from the surface (Hönig and Möbius, 1991). However, as soon as a monolayer floating on the water surface enters the laser focus region, these conditions are changed, reflected light is observed and used to image the monolayer. We used a commercially available microscope (BAM2, Nanofilm Technologie GmbH, Göttingen, Germany), modified with a fiber coupled Argon-Krypton Laser (Innova 70 Spectrum, Coherent). A lateral resolution of $2 \mu \mathrm{m}$ was achieved, sufficient to visualize typically occurring domains in lipid monolayers, which are in the range of $10-100 \mu \mathrm{m}$. Since BAM is very sensitive to changes in the optical parameters of the monolayer, locally confined changes (i.e. domains) in packing or orientation of the molecules are easily monitored. A homemade temperature controlled Teflon trough was used as a film balance. Continuous measurements of the surface pressure were performed with a Wilhelmy balance (Riegler and Kirstein, Mainz, Germany). A VCR and video printer allowed for the experimental documentation.

\section{Results}

Surface pressure area isotherms of the pure SM and POPC phospholipids, as well as binary mixtures of these lipids at different molar ratios, are shown in Fig. 1. The surface pressure-area $(\pi-A)$ isotherm of pure SM indicated a phase transition from the liquid-expanded to

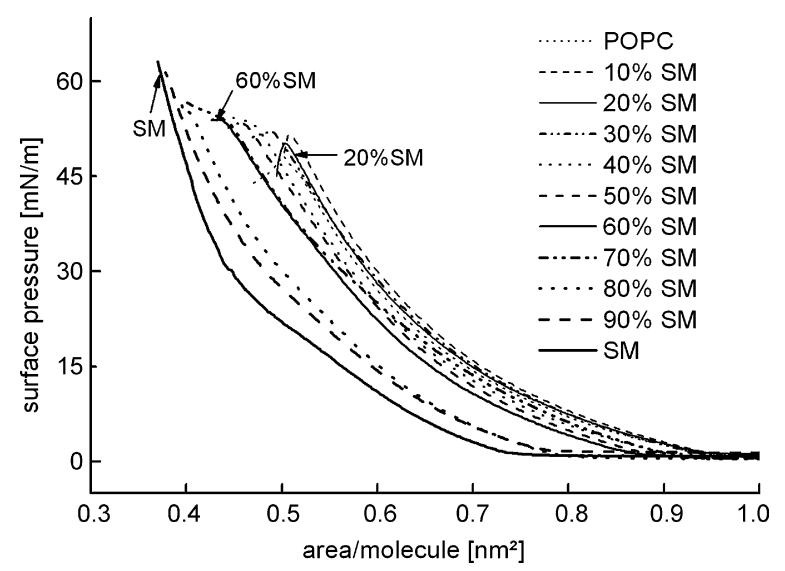

Fig. 1. Surface pressure-area isotherms of SM/POPC recorded at $25^{\circ} \mathrm{C}$ on a subphase of pure water with a compression rate of $0.07 \mathrm{~nm}^{2} /($ molecule min $)$. Symbols in the panel indicate the SM/POPC mixture. 
liquid-condensed state at around $16 \mathrm{mN} / \mathrm{m}$, in agreement with published data (Smaby et al., 1994). Additionally, a second phase transition from the liquid-condensed to a solid phase, characterized by a far steeper slope, can be deduced from the change in the slope of the isotherm around $40 \mathrm{mN} / \mathrm{m}$ (Fig. 1). The appearance of a solid phase is supported by the observation that the Wilhelmy plate started to incline at pressures close to the collapse of the monolayer around $64 \mathrm{mN} / \mathrm{m}$. Furthermore, if the compression was stopped in this surface pressure range, the Wilhelmy plate remained in the inclined position. In contrast to SM, POPC exhibits a typical liquid-expanded behavior consistent with data reported earlier (Smaby et al., 1994). It is evident that sphingomyelin can be compressed to much higher pressures $(>16 \mathrm{mN} / \mathrm{m})$ than POPC before collapse is observed. Moreover, pure POPC was shown to have a larger area per molecule than SM at equivalent surface pressures. This is partly due to the unsaturated fatty acid in position 2 of POPC, which packs less well than the relatively saturated sphingolipid acyl chain content (Binder and Garwisch, 2001) as will be discussed below. These saturated phospholipids allow for more pronounced van der Waals interactions that are effective at short distances. Thus, the closely aligned straight acyl chains result in a higher lateral packing density (Scoville-Simonds and Schick, 2003). In contrast, the disorder induced by unsaturated acyl chains is sufficiently large to inhibit dense packing of adjacent hydrocarbon chains consequently resulting in area expansion (Ali et al., 1991)

The addition of small amounts e.g. 10-20 mol\% of POPC to SM monolayers reduces the collapse pressure by about $10 \%$, while the addition of SM in the same concentration range to POPC increases it to a similar extent. In the intermediate concentration range the collapse pressure shows a linear dependance, increasing slightly with increasing amounts of SM. Even upon significant enlargement of the isotherms no plateau region is observed. Thus it can be concluded that POPC is not squeezed out of these binary mixtures into a separate phase, but is completely dispersed within the SM monolayer: This is also confirmed by the fact that recording of isotherms upon decompression and compression yield reproducible results, demonstrating that no significant amounts of material were lost.

In order to gain further insights into the mixing behavior of POPC and SM, mean molecular areas at different surface pressures were calculated as a function of phospholipid composition (Fig. 2). Deviations from ideal mixing that is represented by the straight lines occurred below $90 \mathrm{~mol} \%$ of SM at all surface pressures. The addition of small amounts of SM (10 mol\%) to a POPC matrix

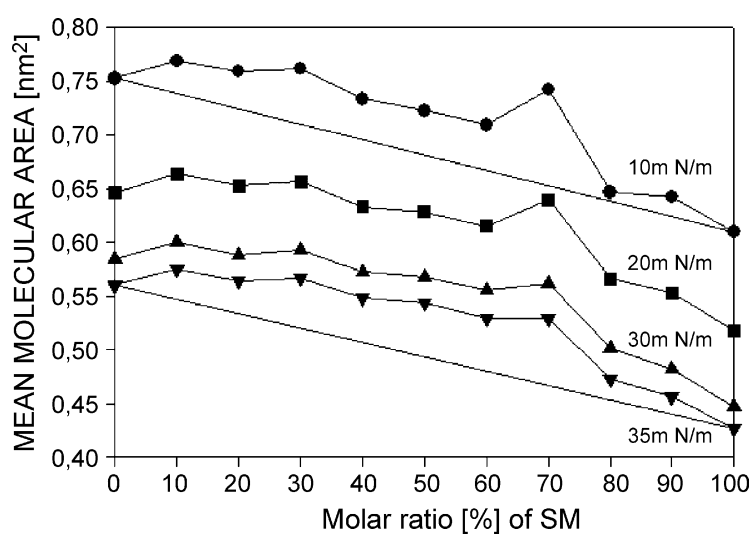

Fig. 2. Mean molecular area vs. phospholipid composition at 10, 20, 30 and $35 \mathrm{mN} / \mathrm{m}$. Ideal additivity of the mean molecular areas is represented by the straight line; surface pressure is indicated in the figure.

leads to an increase of the mean molecular area, which remained almost constant up to $30 \mathrm{~mol} \% \mathrm{SM}$. At higher SM levels, the mean molecular areas decreased but still deviated significantly from an ideal mixing behavior. The largest deviation from an ideal behavior was observed at $70 \mathrm{~mol} \%$ SM. Comparable results for these lipid mixtures were reported by microcalorimetry, where changes in the heat capacity profiles were found below $70 \mathrm{~mol} \%$ SM (Degovics et al., 1997).

Changes in the lateral packing properties may be explained in part by considering the molecular area requirement of the individual component phospholipids. According to the model proposed by Quickenden and Tan (1974), the headgroups of the lipids can be regarded as solid disks as illustrated in the cartoon in Fig. 3. The size of the circles representing POPC and SM is related to their mean molecular area at a pressure of $30 \mathrm{mN} / \mathrm{m}$. Obviously, it is easier for the smaller SM to accommodate the larger POPC molecules in their matrix and hence optimize the lateral packing thereby limiting the unoccupied "free area" (shown in the cartoon in dark grey). In contrast, the packing of the comparably larger POPC is more disturbed by lower amounts of SM that induces more pronounced packing defects in this system (see Fig. 3).

Fig. 4 compares the compressibility of the pure compounds and the mean compressibility of the mixtures according to Eq. (2) whereby the solid line indicates their ideal additivities. In order to avoid ranges in the isotherms exhibiting coexisting phases or phase transitions, we focused on the pressure range between 30 and $35 \mathrm{mN} / \mathrm{m}$ that correlates with the lateral pressure in biomembranes (Marsh, 1996). At $35 \mathrm{mN} / \mathrm{m}, \mathrm{SM}$ exerts a significant effect on the lateral compressibility of the monolayers, and a negative deviation (almost 
(A)

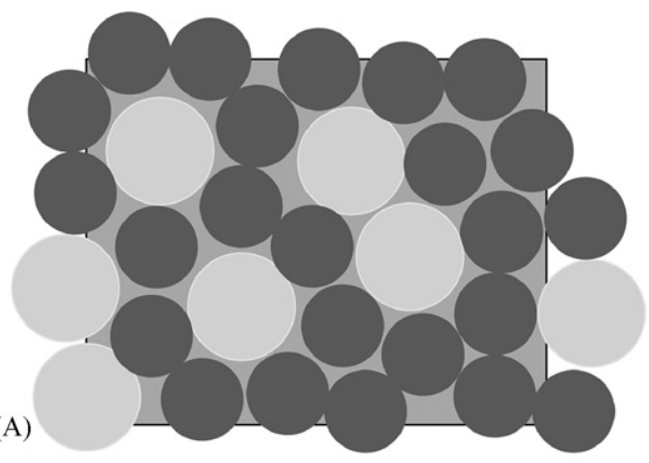

(B)

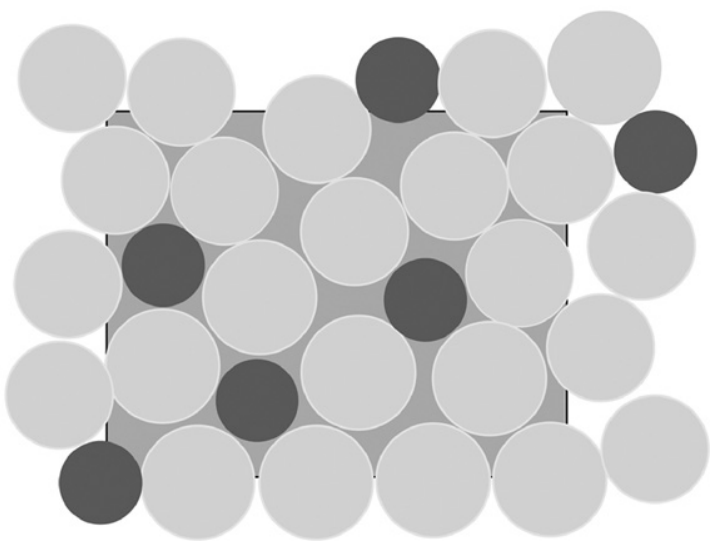

Fig. 3. Cartoon to illustrate potential packing problems between the larger POPC and the smaller area of SM in the monolayer plane. In panel A 24 small disks represent SM (shown in dark) and the 6 larger disks (PC) (shown in light grey) corresponding to a molar ratio of $8: 2$. The free surface, namely the area between the disks is shown in dark grey by the background rectangle. Panel B shows the situation for the opposite molar ratio (2 SM and $8 \mathrm{POPC}$ ). The area of the disks corresponds to the molecular area of SM and POPC, respectively, calculated from the surface pressure-area isotherms at $30 \mathrm{mN} / \mathrm{m}$.

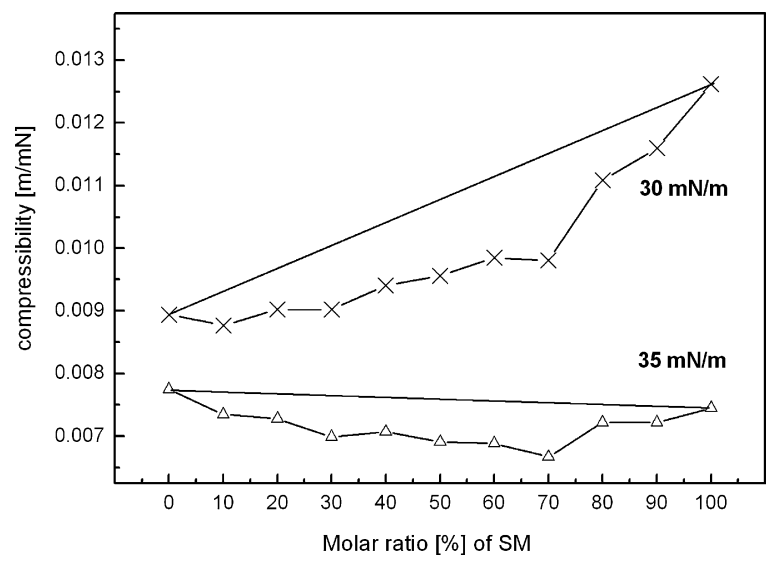

Fig. 4. Mean compressibility vs. phospholipid composition at 30 and $35 \mathrm{mN} / \mathrm{m}$. Ideal additivity of the mean compressibility is represented by the straight line; surface pressure is indicated in the figure.

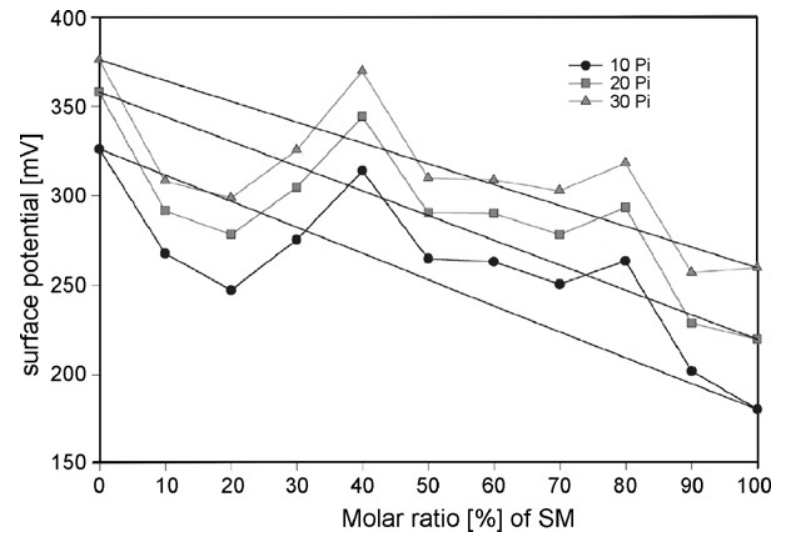

Fig. 5. Mean surface potential measurements vs. phospholipid composition at $10,20,30 \mathrm{mN} / \mathrm{m}$ (pressure is indicated in the figure). Ideal additivity is represented by the straight line.

independent of the molar composition) is observed. Again, the $70 \mathrm{~mol} \% \mathrm{SM}$ mixture exhibited the strongest deviation from ideal mixing.

In Fig. 5, surface potential measurements were recorded and plotted as a function of composition at given surface pressures. In general, the ranges for the potentials of pure SM (up to $280 \mathrm{mV}$ ) in the examined pressure domains were more than $100 \mathrm{mV}$ lower compared to pure POPC (close to $400 \mathrm{mV}$ ). Although the headgroup structure of PC and SM is identical and the side-chain composition is very similar, the chemistry of their backbone differs. In contrast to phosphatidylcholines, sphingomyelins have an additional hydroxyl-group in position 3 and an amide linkage in position 2. Thus the observed pronounced differences in surface potential occur most probably due to the different architecture in the backbone and consequences thereof like the higher probability of sphingomyelin to form intermolecular hydrogen bonds (Ramstedt and Slotte, 2002; Mombelli et al., 2003). Between 10 and $30 \mathrm{~mol} \%$ $\mathrm{SM}$, a significant lower potential than expected from ideal behaviour was observed. In the case of 10 and $20 \mathrm{~mol} \%$ SM the rather small amounts of this lipid influence the mixture to a very high degree and the measured values are closer to the range of pure SM monolayers. On the other hand at 40 and $80 \mathrm{~mol} \%$ SM significantly higher values than expected from the calculations were found, whereby the value exhibited by the mixture containing $40 \mathrm{~mol} \% \mathrm{SM}$ is close to the value of pure PC. Finally, in mixtures containing 50-70 and $90 \mathrm{~mol} \% \mathrm{SM}$ the values are closer to ideal calculations.

Since we demonstrated non-linear deviations of the respective surface areas from ideal mixing, the surface potential had to be correlated to the area of the molecule. Upon compression of the monolayer, changes of the 
surface potential occur that are proportional to the perpendicular component of the dipole moments of the respective amphiphiles. If the conformation or orientation of the lipid and the polarized water remain constant upon area changes the surface dipole moment should be constant. However, deviations from a constant value of the dipole moment are observed (Fig. 6a). Mixtures containing more sphingomyelin exhibit a decreasing change upon reduction of the area. Finally, for pure SM the dipole moment is almost constant over a wide range of compression. The absolute difference between the two pure components is about $500 \mathrm{mD}$. In addition, we compared the actual dipole moments for a given surface area $\left(0.75 \mathrm{~nm}^{2} /\right.$ molecule $)$ as shown in Fig. 6b. This is the region, where POPC reached a surface pressure of about $35 \mathrm{mN} / \mathrm{m}$, which is still well below the collapse pressure of the monolayer. Between 10 and $20 \mathrm{~mol} \%$ SM the dipole moment is lower than calculated for ideal mixing, whereas at $30 \mathrm{~mol} \% \mathrm{SM}$ the dipole moment is close
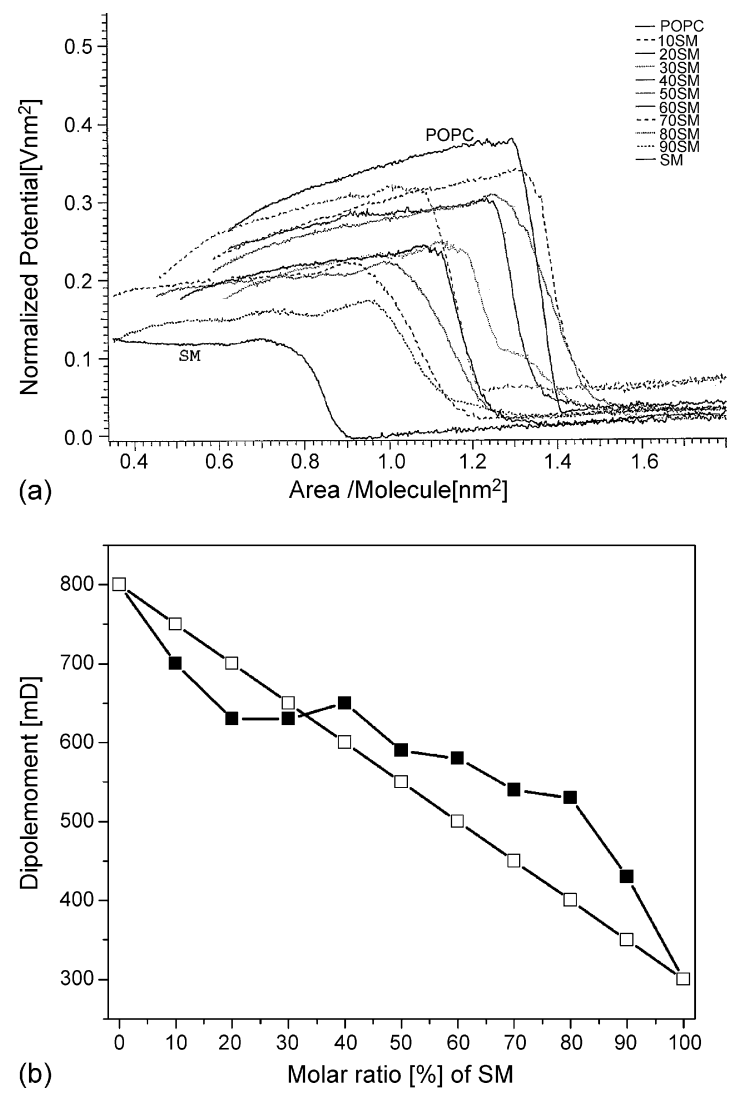

Fig. 6. (a) Vertical dipole moment as a function of the molecular area. SM and POPC content are marked in the graph. (b) Dipole moment taken from plots in panel A at $0.75 \mathrm{~nm}^{2} /$ molecule as a function of the molecular composition. The open symbol (- $\square$-) represents data calculated with the assumption of ideal additivity in the binary mixtures, whereas (-口-) show experimental values. to the one expected for ideal mixing. Above $30 \mathrm{~mol} \%$ the dipole moments are higher than calculated for ideal mixing, in particular the mixture containing $80 \mathrm{~mol} \%$ SM.

In addition to the classical monolayer approach described above, we used a microscopic technique, namely Brewster angle microscopy (BAM) to study the SM/POPC mixtures. Significant differences in the formation of microscopic domains between the two choline phospholipid classes were found. Under our experimental conditions, POPC does not develop macroscopic domains, presumably due to its unsaturated acyl side chain, which result in the formation of a liquid-expanded monolayer throughout the entire surface pressure range. In contrast, SM was observed to form domains that were extremely stable, have a well-defined "finger-like" shape, and often exhibit a six-fold symmetry as shown in Fig. 7a. Moreover, using an analyzer in front of the camera, we observed that these domains exhibit internal organization from the onset of their formation, reflected by the contrast in color of the different sections of a single domain ranging from white to dark grey (Fig. 7a). This is most likely due to a different orientation of the hydrocarbon side chains. Under our experimental conditions, SM domains were already detected at very low surface pressure $(5 \mathrm{mN} / \mathrm{m})$, which stopped growing at around $12 \mathrm{mN} / \mathrm{m}$, and displayed an average size of about $40-50 \mu \mathrm{m}$.

Although biologically relevant domains will be smaller in size, this approach still allows us to investigate the driving forces behind domain formation. Our SM domains form an equidistant super lattice (Fig. 7b) which does not show fusion even at very high surface pressures, where they are forced to contact each other (Fig. 7c). This behavior can be understood in terms of long-range repulsive dipole-dipole interactions (McConnell and de Koker, 1996). Moreover, the SM domains are very stable for a significant period of time, even when the monolayer is rapidly expanded to lower pressures. Upon recompression they will occur at somewhat larger size at slightly lower pressures as compared to the first compression of the SM monolayer. In binary mixtures of SM and POPC similar stable domains were detected between 70 and $90 \mathrm{~mol} \%$ SM. However, the average size was smaller (about $15 \mu \mathrm{m}$ ) and the onset of domain formation was observed at higher surface pressure $(\sim 10 \mathrm{mN} / \mathrm{m})$. The distribution of these domains in the monolayer plane was also not as homogeneous as in pure sphingomyelin monolayers (Fig. 7d). However, the internal organization of domains in such mixtures is the same as in the domains of pure SM. At $60 \mathrm{~mol} \%$ SM additionally a fraction (ca. $10 \%$ ) of smaller round- 

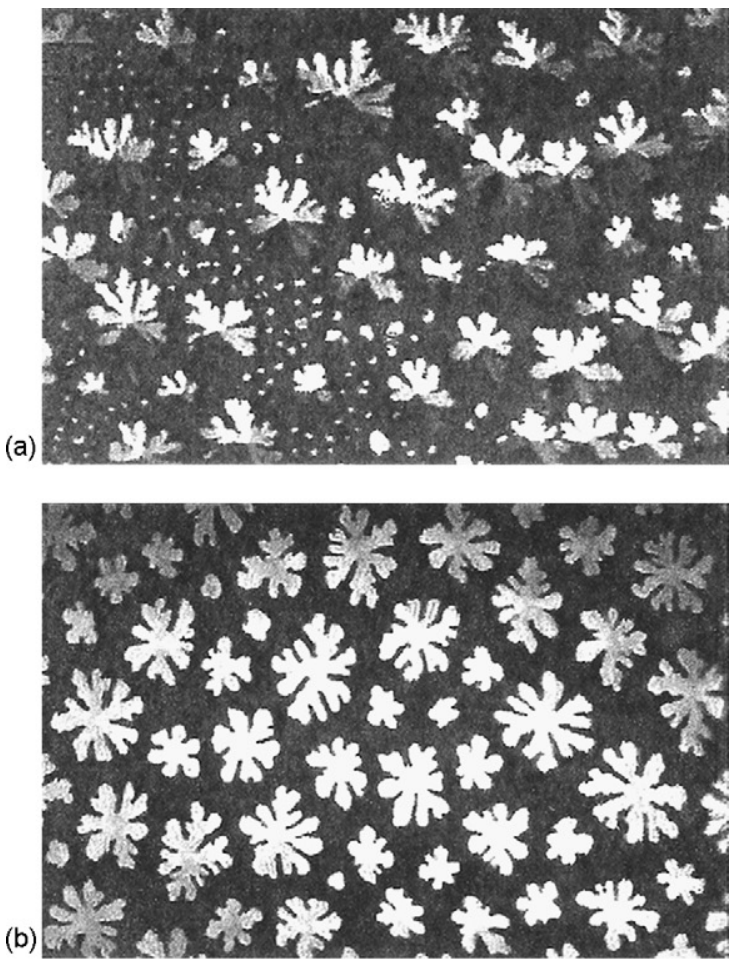

(c)

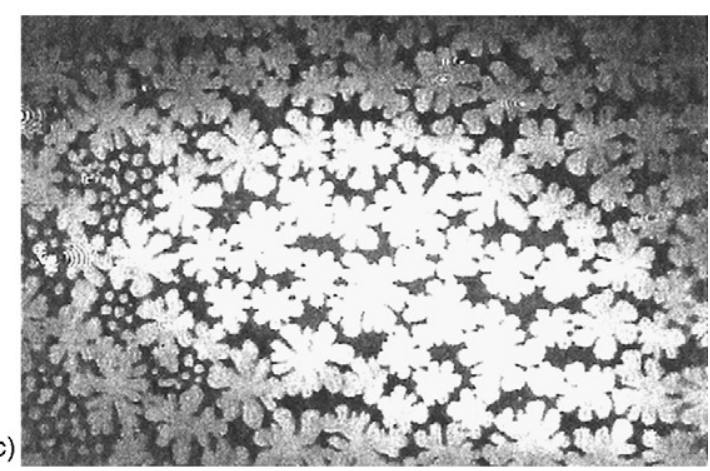

(d)

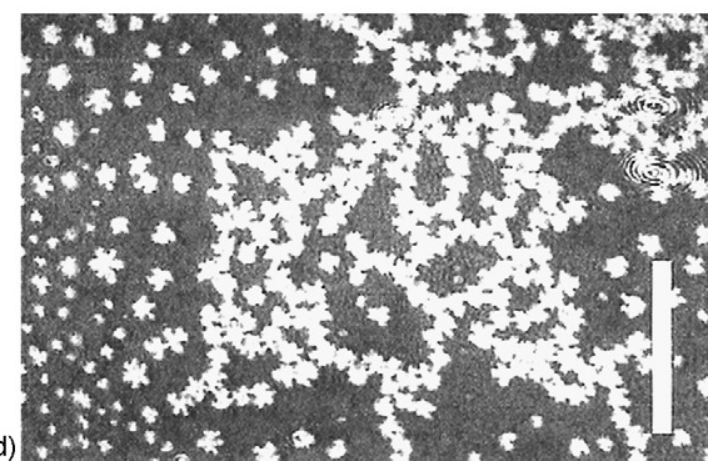

Fig. 7. (Panel a) Brewster angle micrograph of pure sphingomyelin at a surface pressure of $8.2 \mathrm{mN} / \mathrm{m}$ displaying the anisotropic structure of the domains (different color contrast). Liquid-condensed domains appear bright, while liquid-expanded domains remain dark. (Panel b) Brewster angle micrograph of pure sphingomyelin at a surface pressure shaped domains were observed. As well, the SM-like domains exhibited now an average size of $10 \mu \mathrm{m}$ and were less stable in this mixture. They dissolved upon rapid expansion but reforming again upon compression at the same surface pressure. The number of smaller domains with no distinct shape and no detectable internal anisotropy increased upon decreasing the SM content and the onset of domain formation occurred at higher surface pressures. Below $30 \mathrm{~mol} \% \mathrm{SM}$, strongly reduced domain formation was observed and these structures were small and did not grow upon further compression. In general, the Brewster images reflect the characteristics of the monolayer investigations by distinguishing ranges of similar domain formation and shape for systems highly enriched in SM ( $\geq 70 \mathrm{~mol} \%$ $\mathrm{SM})$, an intermediate range between $60 \%$ and $40 \% \mathrm{SM}$ and POPC enriched systems ( $\leq 30 \%$ SM). In Fig. 8, the experimentally determined values for domain size, surface pressure and surface potential as well as molecular area at the onset of domain formation are compared for different monolayers. In panel $\mathrm{A}$, the lateral pressure for the onset of domain formation is shown to increase progressively with a decrease of SM content. While for pure SM first domains are visible at $5 \mathrm{mN} / \mathrm{m}$, at $40 \mathrm{~mol} \%$ SM domains only occur at a surface pressure above $27 \mathrm{mN} / \mathrm{m}$. Concomitantly the average domain size dramatically decreases with decreasing SM content ( $45 \mu \mathrm{m}$ for pure SM and $5 \mu \mathrm{m}$ at $40 \mathrm{~mol} \% \mathrm{SM}$ ). The molecular area, where the domain formation starts, is within a rather narrow range (Fig. $8 \mathrm{~B}$ ) from $0.68 \mathrm{~nm}^{2}$ at $40 \mathrm{~mol} \%$ $\mathrm{SM}$ to $0.77 \mathrm{~nm}^{2}$ at $70 \mathrm{~mol} \% \mathrm{SM}$, i.e. domain formation occurs early on in the compression of the monolayers (see also isotherms in Fig. 1). In the same concentration range the surface potential drops significantly from $310 \mathrm{mV}$ to about $245 \mathrm{mV}$. The faster drop in the surface potential (panel B) on the other hand indicates an early reorientation of the SM that is also confirmed by increasingly flat actual potential isotherms (data not shown).

\section{Discussion}

The present study reports on the mixing behavior and lateral domain organization of SM and PC, which are major components (in varying ratios) of both the outer layer of mammalian cell membranes and monolayers of

of $14.4 \mathrm{mN} / \mathrm{m}$. (Panel c) Brewster angle micrograph of pure sphingomyelin at a surface pressure of $27.9 \mathrm{mN} / \mathrm{m}$. (Panel d) Brewster angle micrograph of a binary mixture containing $70 \mathrm{~mol} \% \mathrm{SM}$ and $30 \mathrm{~mol} \%$ POPC at a surface pressure of $29.9 \mathrm{mN} / \mathrm{m}$. The bar corresponds to $100 \mu \mathrm{m}$. 

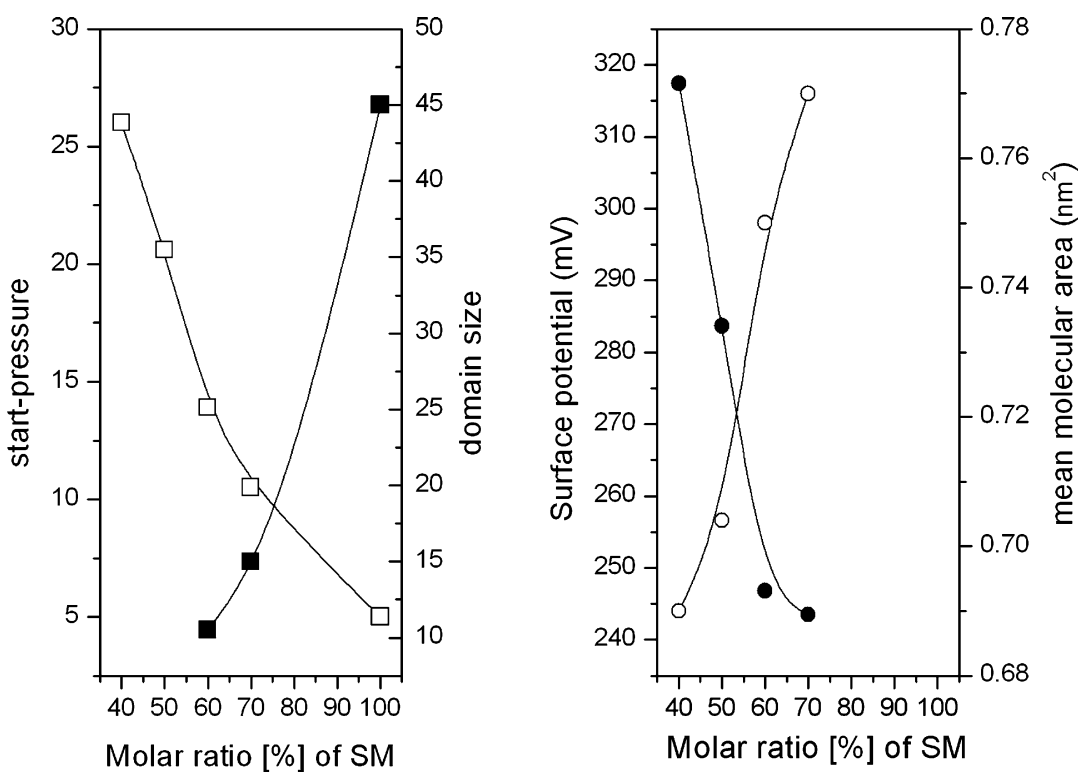

Fig. 8. Panel A illustrates the pressure where domain formation starts (- $\square$-) on the left and the average domain size (- $\square$-) on the right axis as a function of the mol\% SM in the PC/SM mixture. In Panel B, the surface potential in $\mathrm{mV}$ (-)-) on the left and the mean molecular area of these mixtures (- $\bigcirc-$ ) on the right axis are displayed as a function of the $\mathrm{mol} \%$.

lipoproteins. Both lipids have the same phosphocholine headgroup but SM is characterized by an additional free hydroxyl group and an amide-linkage of one side chain. Nevertheless, the first three carbons in the 18-carbon sphingosine base are configurationally equivalent to the carbon atoms of the glycerol of phosphatidylcholine (Ramstedt and Slotte, 2002). As well, the orientation of the hydrocarbon chains is similar, as outlined previously (Smaby et al., 1994).

Monolayers are a well-established technique to investigate lipid mixtures at the air/water interface. Their physical properties are related to those of bilayers (e.g. MacDonald and Simon, 1987) for which an internal pressure of 30-35 mN/m was proposed (Marsh, 1996). Thus, monolayers composed of binary mixtures of SM and PC were prepared, which allowed controlling of the composition with high accuracy.

Analysis of the surface pressure-area isotherms revealed a non-ideal mixing of both components over a large composition range, similar to what has been reported for mixtures of galactosylceramides and phosphatidylcholines (Ali et al., 1991). Calculation of the mean molecular areas for pure SM and POPC, as well as their respective mixtures, demonstrated that the binary phospholipid mixtures showed an expansion of their respective areas over a large range $(10-80 \mathrm{~mol} \% \mathrm{SM})$ as was indicated by the positive deviation of the mean molecular areas from the ideal values. These observations differ from what was observed with cholesterol, which is known to exhibit a condensing effect on most phosphatidylcholines and sphingomyelins (Hao and Chen, 2001).

Inspection of the profile of the isotherms revealed that demixing of the phospholipids and macroscopic phase separation does not occur, since no plateau regions were observed at the POPC collapse pressure indicative for an earlier collapse of separated POPC domains. Our results agree with previously published calorimetric and X-ray data (Maulik and Shipley, 1996; Degovics et al., 1997). While lower amounts of POPC (up to about $20 \mathrm{~mol} \%$ ) can be incorporated into the SM matrix in an ideal manner, higher amounts induce non-ideal packing. The incorporation of low amounts of SM into a POPC monolayer gave similar results but here even at $10 \mathrm{~mol} \% \mathrm{SM}$ the mean molecular area of the lipids in the monolayer was largely expanded. An effect of low amounts of SM on the two-dimensional lateral packing was also reported for the oxidation rate of cholesterol in a ternary mixture consisting of phosphatidylcholine, sphingomyelin and cholesterol (Mattjus and Slotte, 1994, 1996).

According to a simple model (Quickenden and Tan, 1974) lipids are compared to solid disks of uniform size covering a given surface that is progressivly reduced to mimic compression. It has to be noted that only geometrical considerations are included and other interactions of e.g. electrostatic nature, which can have additional impact on such a system, were ignored. After a degree of compression comparable to monolayer data, there were always areas detected in the plane, where defects occur, even at very high compression mimicking a solid phase. 
This means that the discs are not packed absolutely ideal and homogeneously. Hence, it is evident that the ratio of two compounds differing significantly in their molecular cross sectional areas like SM and POPC will exhibit even more packing problems and thus more packing defects. Fig. 3 clearly illustrates that the smaller SM has a stronger impact on the packing of the larger POPC discs and thus packing defects are induced. The extent of this effect will depend on the actual molar ratios of the mixture. The effect is even stronger than in this small cartoon, when a larger number of disks is considered, since packing problems can affect many disks beyond the first layer of POPC around a smaller SM.

Although no macroscopic phase separation in the binary lipid mixture is observed, the molar ratio of the two lipids is obviously important and can give rise to microscopic differences in their lateral organization, which might be amplified by the interaction with other membrane constituents. One can envision that the large number of lipids found in cells may impose a variety of localized packing problems. Such packing defects have been proposed to occur at the boundaries of membrane lipid domains (Sackmann, 1983) and may play a role for biologically relevant processes like the interaction of peptides with membranes. Preferential peptide insertion and accumulation in such defect lines was proposed (Colotto et al., 1993). In addition, the lipid composition of LDL (70\% PC and 30\% SM) (Sommer et al., 1992) is in the region where we find significant deviations from ideal packing which maybe be physiologically relevant by faciliating the insertion of the apoprotein $\mathrm{B}$ into the LDL particle.

The lateral compressibility of the mixed lipid monolayers was significantly affected by sphingomyelin in particular at higher surface pressures, where a negative deviation almost independent of the molar composition of the mixtures is observed. Changes in lateral compressibility may have a direct impact on interfacial enzymatic activity (Marsh, 1996). Furthermore, domain formation, as discussed below, might contribute to discontinuities in the lateral packing pressure that should be reflected in physiological functions. Thus, the varied content of $\mathrm{PC}$ and SM and the resulting differences in packing and rigidity in terms of their compressibility might affect their distinct role in the membrane as discussed by Marsh (1996), who suggested that a range of functional properties in a membrane are determined by the lateral compressibility of the membrane.

The quantification of the main interactions between molecules in membranes are still unsolved, since the evaluation of the local electrostatic properties near interfaces are a basic problem (McConnell and de Koker,
1996). The respective value of the dielectric constant of water changes from about $80 \mathrm{D}$ in bulk water to about $2 \mathrm{D}$ in a PC membrane interface (Brockman, 1994). The surface potential values for the pure components PC and SM, though structurally related, differ significantly by about $120 \mathrm{mV}$ in agreement with earlier data (Ali et al., 1991). Since biomembranes contain lipids and proteins carrying formal charges, relatively fixed in their position, the existing dipoles can only be partially compensated by water dipoles or solution electrolytes and thus give rise to a complex array of electric fields (Brockman, 1994). Such differences may have a wide impact on biological processes based on differences in the surface potential or local charge distribution in the vicinity of such lipid domains. Our data clearly show that even binary mixtures of major mammalian lipids exhibit complex mixing behavior that affect packing density and the resulting surface potential. The two major membrane lipid mixtures as shown in this study exhibit different surface potentials that might affect their affinity and interaction with (apo)proteins, in addition to the packing problems discussed above. This is emphasized by the fact that distinct behavior of PC and SM in some lipoprotein systems was reported as preferential interactions of apoproteins with phosphatidylcholine in LDL (Sommer et al., 1992; Murphy et al., 1997).

Upon monolayer compression a reorientation of molecular dipoles, changes in the hydration of the polar headgroups (Vollhardt et al., 1978) and reorganisation of the vicinal water layers (Bockris and Jeng, 1990) occur. Thus, it is import to analyze the surface potential as a function of the surface area which is shown in Fig. 6a that illustrates the vertical component of the dipole moment. The very flat slopes of the high SM systems (e.g. 90 and $100 \mathrm{~mol} \%$ ) mean that there are no relevant dipole reorientations taking place upon compression. This is in agreement with an early alignment of the molecules upon domain formation that does not change upon further compression due to the strong intermolecular interactions, while in the POPC enriched part (e.g. 0-30 mol\% $\mathrm{SM})$ there is a constant decrease. Explanations for this behaviour as proposed by Schumann (1990) are a changing distribution of molecular conformations, changing dipole orientations due to steric hinderances or interactions and variations of the local dielectric constant. Interestingly, in highly POPC dominated monolayers no significant domain formation or domain growth are observed (see below). In Fig. 6b, the dipole moment for these mixtures at a constant area $\left(0.75 \mathrm{~nm}^{2} /\right.$ molecule $)$ are plotted against the molar ratio. This is the respective area, where POPC reached a surface pressure of about $35 \mathrm{mN} / \mathrm{m}$ which is close to the proposed lateral pressure 
in biological membranes (Marsh, 1996) but still away from the collapse pressure of the monolayers. At low SM content (10 and $20 \mathrm{~mol} \% \mathrm{SM}$ ) the dipole moment is decreased as compared to additivity and increased at higher SM content, in particular at $80 \mathrm{~mol} \%$ SM suggesting that the different molar $\mathrm{PC} / \mathrm{SM}$ composition molar of mamamlian biomembranes will also result with significant difference in dipole moments that may impact binding or insertion affinities of ligands or proteins into these matrices.

Furthermore, an increase of the lamellar repeat distance upon addition of PC to SM was shown by X-ray diffraction studies (Degovics et al., 1997; Koschuch, 2002) and was also observed for ternary SM/POPC/CHOL (Koschuch, 2002). Global modelling of the X-ray data showed that this increase is due to an increase of both bilayer thickness and intra-lamellar water, e.g. at $20^{\circ} \mathrm{C}$ a bilayer thickness of $4.6 \mathrm{~nm}$ was reported for pure POPC (Rappolt et al., 2004) as compared to $5.5 \mathrm{~nm}$ for an equimolar mixture SM/POPC containing $1 \mathrm{~mol} \%$ cholesterol (Pabst et al., 2003). A difference of about $1 \mathrm{~nm}$ in bilayer thickness was also reported between detergent soluble and detergent resistant membranes (Gandhavadi et al., 2002). This implies that such SM domains, in addition to tighter packing of the acyl chain, are structures that protrude from the plane. These lipid domains, characterized by an increased bilayer thickness, will most likely also exhibit localized differences in the dipole moment in contrast to the bulk membrane and thus may reflect preferential binding or interaction sites on the membrane surface.

Brewster angle microscopy demonstrated microscopic domain formation for mixtures containing more than $30 \mathrm{~mol} \% \mathrm{SM}$, whereby shape, size and appearance were strongly dependent on the lipid composition. Domains formed by pure SM were characterized by a finger-like shape, predominantly consisting of six main branches, and exhibited the largest size occurring already at low surface pressure (Fig. 7a and b). As seen in Fig. 7a these domains exhibit internal anisotropy indicating a high degree of internal order and tilted chains for the six main branches. The fact that these structures exhibit internal optical anisotropy indicates long-range order of the hydrocarbon chains (Vollhardt, 1996; Johann et al., 2000). Only mixtures with more then $70 \mathrm{~mol} \%$ of SM would show such domains that grew to a limiting surface pressure. These domains were very stable and did not fuse even at very high surface pressure (Fig. 7c). This behavior is more pronounced than observed for other lipid domains (e.g. DMPE), where such domains would fuse at high surface pressure (Hönig and Möbius, 1991).
Another indication for the stability of these domains is the fact that if the monolayers are decompressed and compressed again, domains appear at very low lateral pressure, indicating the persistence of seed crystals in these films. With increasing POPC content the domain decreased in size (Fig. 7d) and adopted rounder shapes concomitantly shifting the onset of their formation to higher surface pressure. Such a change in the domain shape was also reported for glycerol esters and glycerol amides (Gehlert et al., 1998). It was suggested that the differences in shape are due to hydrogen bonding and a more condensed phase of the amide species.

Presumably, the hydrogen bonding capacity of sphingomyelin and its longer and closely aligned straight acyl chains results in a higher lateral packing density resulting in more pronounced van der Waals interactions operating at short distances (Scoville-Simonds and Schick, 2003). Fluorescence anisotropy measurements confirmed a more rigid packing of the SM matrix as compared to a PC system (Prenner et al., 1993). In addition, recent NMR data showed an ordering effect due to SM in PC/SM mixtures (Steinbauer et al., 2003). Assuming that larger proportions of SM are involved in the formation of rigid domains, preferential apoprotein interaction with the more fluid phase or the fluid PC component is reasonable, as discussed above for apoprotein interactions in LDL (Sommer et al., 1992; Murphy et al., 1997).

These SM or SM enriched domains could be relevant for lipoprotein architecture as discussed above and were proposed to play a role in blood platelets activation (Bodin et al., 2003). In this context, it is interesting to consider the fact that the SM content of atherosclerotic lesion is about $80 \%$ (Schissel et al., 1998) and this might suggest a more rigid environment that will concomitantly affect physiological functions.

Finally, our data demonstrate the formation of very stable and highly organized SM domains in pure SM and SM enriched matrices that will probably exhibit local differences in rigidity and surface potential. The overview of the experimental parameters in Fig. 8 support the conclusion that SM plays a key role in the formation and stability of the observed domains.

The average size increased and the on-set pressure of domain formation decreased when more SM is in the system (panel A). Concomittantly, there is a significant drop in surface potential and the lower onset pressure is also reflected in the larger mean molecular areas at domain formation (panel B).

All parameters discussed here were observed without cholesterol and thus sterol independent rafts should be likely as were reported for the kidney- and intestinal brush border membrane models (Milhiet et al., 2002). 
We suggest that such observed stable domains could be initial building blocks seeding so-called membrane rafts. They could be formed by different lipids depending on the actual system under investigation, with or without a direct contribution from cholesterol. The rigid packing propensity of sphingosine based lipids and their ability to form extensive hydrogen bonds seem to be important factors for their formation and stability. Furthermore, SM enriched domains were found in the Golgi (Gkantiragas et al., 2001), ceramide-rich rafts were proposed to facilitate CD40 clustering (Grassme et al., 2002), whereas ganglioside enriched domains were reported in human fibroblasts (Nagatsuka et al., 2003) and finally phosphatidylglucoside based domains in HL60 cells (Chigorno et al., 2000).

Considering lipid acyl chain diversity and the resulting large number of lipid subspecies in a multitude of possible packing defects, surface potential "islands" and lateral domains of varying sizes in biomembranes can be envisioned. Domains will exhibit differences in rigidity, stability, and kinetics of formation (or disintegration) and all three factors will offer differential or preferential interaction or insertion sites for various proteins. This multitude of possible structures and features facilitates to comprehend the numerous areas, where a role for lipid rafts has been proposed. Ultimately, a better understanding of the raft structure and function should serve as an avenue to develop novel treatments for diseases like virus infections, atheroscleosis or neurodegenerative disorders (Barenholz, 2004).

\section{Acknowledgements}

The authors thank Werner Zeiss and Daniela B. Spohn for expert technical assistance, and Elke LohmeierVogel for critically reading the manuscript. EJP holds an Alberta Heritage Foundation for Medial Research (AHFMR) scholar award. Financial support was also provided by AHFMR and the European Biology Organization (ASTF 7662 at the MPI-BPC to EJP). Technical support was kindly provided by Nanofilm Technologie $\mathrm{GmbH}$, Göttingen, Germany.

\section{References}

Ali, S., Brockman, H.L., Brown, R.E., 1991. Structural determinants of miscibility in surface films of galactosylceramide and phosphatidylcholine: effect of unsaturation in the galactosylceramide acyl chain. Biochemistry 30, 11198-11205.

Ali, S., Smaby, J.M., Brockman, H.L., Brown, R.E., 1994. Cholesterol's interfacial interactions with galactosylceramides. Biochemistry 33, 2900-2906.
Anderson, R.G.W., Jacobson, K., 2002. Cell biology: a role for lipid shells in targeting proteins to caveloae, rafts and other lipid domains. Science 296, 1821-1825.

Barenholz, Y., Gatt, S., 1982. Sphingomyelin: metabolism, chemical synthesis and chemical properties. In: Hawthorne, J.N., Ansell, G.B. (Eds.), Phospholipids. Elsevier Biomedical Press, NY, pp. 129-177.

Barenholz, Y., 2004. Sphingomyelin and cholesterol: from membrane biophysics and rafts to potential medical applications. Sub-Cellular Biochem. 37, 167-215.

Bartlett, G.R., 1959. Phosphorus assay in column chromatography. J. Biol. Chem. 234, 466-468.

Binder, H., Garwisch, K., 2001. Effect of unsaturated lipid chains on dimensions, molecular order and hydration of membranes. J. Phys. Chem. B 105, 12378-12390.

Bockris, J.O.M., Jeng, K.T., 1990. Waters structures at interfaces-the present situation. Adv. Colloid Interf. Sci. 33, 1-54.

Bodin, S., Tronchere, H., Payrastre, B., 2003. Lipid rafts are critical membrane domains in blood platelet activation processes. Biochim. Biophys. Acta 1610, 247-257.

Brockman, H.L., 1994. Dipole potential of lipid membranes. Chem. Phys. Lipids 73, 57-79.

Brown, D.A., London, E., 1998. Functions of lipid rafts in biological membranes. Annu. Rev. Cell Dev. Biol. 14, 111-136.

Cesar, J.M., Vecino, A., Perez-Vasqueo, M., Navarro, J.L., 1993. Phospholipid determination in platelets, plasma and red cells of patients with chronic myeloproliferative disease. Eur. J. Haematol. 50, 234-236.

Chigorno, V., Palestini, P., Sciannamblo, M., Dolo, V., Pavan, A., Tettamanti, G., Sonnino, S., 2000. Evidence that ganglioside enriched domains are distinct from caveolae in MDCK II and human fibroblast cells in culture. Eur. J. Biochem. 267, 4187-4197.

Colotto, A., Kharakoz, D.P., Lohner, K., Laggner, P., 1993. Ultrasonic study of melittin effects on phospholipid model membranes. Biophys. J. 65, 2360-2367.

Degovics, G., Latal, A., Prenner, E., Kriechbaum, M., Lohner, K., 1997. Structure and thermotropic behavior of mixed choline phospholipid model membranes. J. Appl. Cryst. 30, 776-780.

Gandhavadi, M., Allende, D., Vidal, A., Simon, S.A., McIntosh, T.J., 2002. Structure, composition, and peptide binding properties of detergent soluble bilayers and detergent resistant rafts. Biophys. J. $82,1469-1482$.

Gehlert, U., Weidemann, G., Vollhardt, D., Brezesiniski, G., Wagner, R., Möhwald, H., 1998. Relating domain morphology and lattice structure in monolayers of glycerol amide lipids. Langmuir 14, 2112-2118.

Giocondi, M.-C., Boichot, S., Plenat, T., Le Grimellec, C., 2004. Structural diversity of sphingomyelin microdomains. Ultramicroscopy 100, 135-143.

Gkantiragas, I., Brugger, B., Stuven, E., Kaloyanova, D., Li, X.Y., Lohr, K., Lottspeich, F., Wieland, F.T., Helms, J.B., 2001. Sphingomyelin-enriched microdomains at the Golgi complex. Mol. Biol. Cell. 12, 1819-1833.

Grassme, H., Jendrossek, V., Bock, J., Riehle, A., Gulbins, E., 2002. Ceramide-rich membrane rafts mediate CD40 clustering. J. Immunol. 168, 298-307.

Hao, Y.H., Chen, J.W., 2001. Influence of cholesterol on the biophysical properties of the sphingomylein/DOPC binary system. J. Membr. Biol. 183, 85-92.

Harder, T., 2003. Formation of functional cell membrane domains: the interplay of lipid- and protein-mediated interactions. Philos. Trans. R. Soc. London, Ser. B: Biol. Sci. 358 (1433), 863-868. 
Henon, S., Meunier, J., 1991. Microscope at the Brewster angle: direct observation of first-order phase transitions in monolayers. J. Rev. Sci. Instrum. 62, 936-939.

Hönig, D., Möbius, D., 1991. Direct visualization of monolayers at the air-water interface by Brewster angle microscopy. J. Phys. Chem. $95,4590-4592$.

Johann, R., Vollhardt, D., Möhwald, H., 2000. Faceting of monolayer domains. Colloid Polym. Sci 278, 104-113.

Keller, S.L., 2003. Miscibility transitions and lateral compressibility in liquid phases of lipid monolayers. Langmuir 19, 1451-1456.

Koschuch, R., 2002. Development of a moveable SAXS-system for high-throughput-screening and structure determination of a threecomponent model membrane. Thesis, University of Technology, Graz, Austria.

Kuhn, H., Möbius, D., 1993. Investigations of surfaces and interfaces. In: Rossiter, B.W., Baetzold, R.C. (Eds.), Physical Methods of Chemistry Series, vol. IXB. John Wiley \& Sons, UK, p. 375.

Laggner, P., 1981. Lateral diffusion of lipids in sarcoplasmic reticulum membranes is area-limited. Nature 294, 393-394.

London, E., 2002. Insight into lipid raft structure and formation from experiments in model membranes. Cur. Opin. Struc. Biol. 12, 480-486.

MacDonald, R.C., Simon, S.A., 1987. Lipid monolayer states and their relationship to bilayers. Proc. Natl. Acad. Sci. U.S.A. 84, 4089-4093.

Marsh, D., 1996. Lateral pressure in membranes. Biochim. Biophys. Acta 1286, 183-223.

Mattjus, P., Slotte, J.P., 1994. Availability for enzyme-catalyzed oxidation of cholesterol in mixed monolayers containing both phosphatdiylcholine and sphingomyelin. Chem. Phys. Lipids 71, 73-81.

Mattjus, P., Slotte, J.P., 1996. Does cholesterol discriminate between sphingomyelin and phosphatidylcholine in mixed monolayers containing both phospholipids. Chem. Phys. Lipids 81, 69-80.

Maulik, P.R., Shipley, G.G., 1996. N-Palmitoyl sphingomyelin bilayers: structure and interactions with cholesterol and dipalmitoylphosphatidylcholine. Biochemistry 35, 8025-8034.

McConnell, H.M., de Koker, R., 1996. Equilibrium thermodynamics of lipid monolayer domains. Langmuir 12, 4897-4904.

Milhiet, P.E., Domec, C., Giocondi, M.-C., Van-Mau, N., Heitz, F., Le-Grimellec, C., 2001. Domain formation in models of the renal brush border membrane outer leaflet. Biophys. J. 81, 547-555.

Milhiet, P.E., Giocondi, M.C., Le Grimellec, C., 2002. Cholesterol is not crucial for the existence of microdomains in kidney brushborder membrane models. J. Biol. Chem. 277, 875-878.

Mombelli, E., Morris, R., Taylor, W., Fraternali, F., 2003. Hydrogenbonding propensities of sphingomyelin in solution and in a bilayer assembly: a molecular dynamics study. Biophys. J. 84, 15071517.

Murphy, H.C., Korpela, M.A., White, J.J., Raoof, A., Bell, J.D., Barnard, M.L., Burns, S.P., Iles, R.A., 1997. Evidence for distinct behavior of phosphatidylcholine and sphingomyelin at the low density lipoprotein surface. Biochem. Biophys. Res. Commun. 23, 733-737.

Nagatsuka, Y., Hara-Yokoyama, M., Kasama, T., Takekoshi, M., Maeda, F., Ihara, S., Fujiwara, S., Ohshima, E., Ishii, K., Kobayashi, T., Shimizu, K., Hirabayashi, Y., 2003. Carbohydratedependent signaling from the phosphatidylglucoside-based microdomain induces granulocytic differentiation of HL60 cells. Proc. Natl. Acad. Sci. U.S.A. 100, 7454-7459.

Ohanian, J., Ohanian, V., 2001. Sphingolipids in mammalina cell signalling. Cell. Mol. Life Sci. 58, 2053-2068.
Pabst, G., Koschuch, R., Pozo-Navas, B., Rappolt, M., Lohner, K., Laggner, P., 2003. Structural analysis of weakly ordered membrane stacks. J. Appl. Cryst. 36, 1378-1388.

Prenner, E., Hermetter, A., Landl, G., Stütz, H., Kauffmann, H.F., Kungl, A., 1993. Fluorescence lifetime distributions of various phospholipids labeled with covalently bound diphenylhexatriene in the erythrocyte ghost membrane. J. Phys. Chem. 97, 27882792.

Quickenden, T.I., Tan, G.K., 1974. Random packing in two dimensions and the structure of monolayers. J. Colloid Interf. Sci. 48, 382393.

Radhakrishnan, A., Anderson, T.G., McConnell, H.M., 2000. Condensed complexes, rafts, and the chemical activity of cholesterol in membranes. Proc. Natl. Acad. Sci. U.S.A. 97, 12422-12427.

Ramstedt, B., Slotte, J., 2002. Membrane properties of sphingomyelins. FEBS Lett. 531, 33-37.

Rappolt, M., Laggner, P., Pabst, G., 2004. Structure and elasticity of phospholipid bilayers in the $L_{\alpha}$ phase: a comparison of phosphatidylcholine and phosphatidylethanolamine membranes. Recent Res. Dev. Biophys. 3, 363-394.

Rinia, H.A., Snel, M.M.E., van-der-Eerden, J.P.J.M., de Kruijff, B., 2001. Visualizing detergent resistant domains in model membranes with atomic force microscopy. FEBS Lett. 501, 92-96.

Sackmann, E., 1983. Physical foundations of the molecular organization and dynamics of membranes. In: Hoppe, W., Lohmann, W., Markl, H., Ziegler, H. (Eds.), Biophysics. Springer-Verlag, New York, p. 425.

Saez-Cirion, A., Nir, S., Lorizate, M., Agirre, A., Cruz, A., PerezGil, J., Nieva, J.L., 2002. Sphingomyelin and cholesterol promote HIV-1 gp41 pretransmembrane sequence surface aggregation and membrane restructuring. J. Biol. Chem. 277, 21776-21785.

Schissel, S.L., Jiang, X.C., Tweedie-Hardman, J., Jeong, T.S., Camejo, E.H., Najib, J., Rapp, J.H., Williams, K.J., Tabas, I., 1998. Secretory sphingomyelinase, a product of the acid sphingomyelinase gene, can hydrolyze atherogenic lipoproteins at neutral $\mathrm{pH}$ : implications for atherosclerotic lesion development. J. Biol. Chem. 273, 2738-2746.

Schumann, D., 1990. Electrical properties of adsorbed or spread films: the effective value of permittivities in the Helmholtz equation (plane distribution of point dipoles). J. Colloid Interf. Sci. 134, $152-160$.

Scoville-Simonds, M., Schick, M., 2003. Theory of the effect of unsaturation on the main-chain transition. Phys. Rev. E 67(1), art. no. 011911.

Simons, K., Ikonen, E., 1997. Functional rafts in cell membranes. Nature 387, 569-572.

Simons, K., Toomre, D., 2000. Lipid rafts and signal transduction. Nat. Rev. 1, 31-41.

Simons, K., Vaz, W.L.C., 2004. Model systems, lipid rafts and cell membranes. Annu. Rev. Biophys. Biomol. Struct. 33, 269-295.

Smaby, J.M., Brockman, H.L., Brown, R.E., 1994. Cholesterol's interfacial interactions with sphingomyelins and phosphatidylcholines: hydrocarbon chain structure determines the magnitude of condensation. Biochemistry 33, 9135-9142.

Sommer, A., Prenner, E., Gorges, R., Stütz, H., Grillhofer, H., Kostner, G.M., Paltauf, F., Hermetter, A., 1992. Organization of phosphatidylcholine and sphingomyelin in the surface monolayer of low density lipoprotein and lipoprotein(a) as determined by timeresolved fluorometry. J. Biol. Chem. 267, 24217-24222.

Steinbauer, B., Mehnert, T., Beyer, K., 2003. Hydration and lateral organization in phospholipid bilayers containing sphingomyelin: a 2H-NMR study. Biophys. J. 85, 1013-1024. 
Van-Mau, N., Misse, D., Le-Grimellec, C., Divita, G., Heitz, F., Veas, F., 2000. The SU glycoprotein 120 from HIV-1 penetrates into lipid monolayers mimicking plasma membranes. J. Membr. Biol. 177, 251-257.

Veatch, S.-L., Keller, S.L., 2003. Separation of liquid phases in giant vesicles of ternary mixtures of phospholipids and cholesterol. Biophys. J. 85, 3074-3083.

Vogel, V., Möbius, D., 1988a. Local surface potential and electric dipole moments of lipid monolayers: contributions of the water/lipid and the lipid/air interfaces. J. Colloid Interf. Sci. 126, 408-420.

Vogel, V., Möbius, D., 1988b. Hydrated polar groups in lipid monolayers: effective local dipole moments and dielectric properties. Thin Solid Films 159, 73-81.

Vollhardt, D., Wüstneck, R., Zastrow, L., 1978. Studies of dynamic behavior of spread monolayers at interface of air-aqueous solution- surface potential of spread octadecanol, octadecanoic acid, and Na-octadecyl sulfate. Coll. Polym. Sci. 256, 983-990.

Vollhardt, D., 1996. Morphology and phase behavior of monolayers. Adv. Colloid Interf. Sci. 64, 143-171.

Wang, T.-Y., Silvius, J.R., 2000. Different sphingolipids show differential partitioning into sphingolipid/cholesterol-rich domains in lipid bilayers. Biophys. J. 79, 1478-1489.

Wang, T.-Y., Leventis, R., Silvius, J.R., 2001. Partitioning of lipidated peptides sequences into liquid-ordered domains in model and biological membranes. Biochemistry 40, 13031-13040.

Xu, X., Bittman, R., Duportail, G., Heissler, D., Vilcheze, C., London, E., 2001. Effect of the structure of natural sterols and sphingolipids on the formation of ordered sphingolipid/cholesterol domains (rafts): comparison of cholesterol to plant, fungal and diseaseassociated sterols, and comparison of sphingomyelin, cerebrosides and ceramide. J. Biol. Chem. 276, 33540-33546. 